\title{
Application Status of Face Recognition Products for Chinese Public Security
}

\author{
Zihao Zhang \\ People's Public Security University of China, Beijing Daxing 100076 \\ 615791251@qq.com
}

\begin{abstract}
Keywords: Face recognition product; non-contact; public security criminal investigation; face attribute analysis

Abstract: As one of the bio-metric identification technologies, face recognition is widely used in the field of public security due to its non-contact collection characteristics and good user acceptance. Currently, mature and typical face recognition products companies on the market include: Google companies and Facebook companies in the United States; Yitu Technology, Beijing Kuangshi Technology Co., Ltd., Jinshi Technology Co., Ltd., Zhongke Vision Co., Ltd.,Yuncong Technology Co., Ltd., Guangzhou Pixel Co., Ltd., Haixin Kejin Co., Ltd., Shangtang Technology Co., Ltd.in China and so on. They have in-depth cooperation with the public security organs, providing a variety of face recognition products, which makes the power of face recognition in the public security work to the fullest. Through the investigation, this paper sorts out the face recognition products of several face recognition technology companies that cooperate with public security criminal investigation in the market and related products applicable to public security criminal investigation work.
\end{abstract}

\section{The Basic Category of Face Recognition Technology}

Face recognition technology refers to the technique of comparing and confirming one or more people in an image using an existing face database given a still or moving image. There are three distinct stages in the development of face recognition. Since the beginning of the 1960s, the research work of face recognition has experienced more than 50 years of development, and has became one of the most popular research contents in the field of image analysis and recognition [1], while China's face recognition research started in the 1980s .Although China start late, it has achieved a lot of research results, and some companies and products have reached the world advanced level.

In recent years, the rapid development of bio-metrics technology has become a mature means of identification and has received widespread attention. Face recognition, by virtue of its direct, friendly and convenient features, has become the most accepted bio metric method, and has become one of the most active research areas in bio-metrics [2].

With the development of science and technology and the popularity of surveillance, the role of face recognition in public security criminal investigation work has become increasingly important. Through the face recognition to determine the identity of the offenders and conduct search and tracking, the means of inserting the face information into the library to crack other cases have became weapons for the public security organs to crack down the crimes .Therefore, the understanding and investigation of the face 
recognition products for public security criminal investigation and their current status is of great significance to the development of public security work [3].

\section{Face Recognition Products and Application Status of Public Security}

\subsection{Yitu Technology Co., Ltd. and Blink Intelligent Security Platform}

Shanghai Yitu Network Technology Co., Ltd.'s blinking portrait platform technology is widely used in the public security system's identity screening, synthetic pursuit, detection and resolution, service for people's livelihood and other businesses. Through the system, the public security organs can quickly judge the consistency of the object and the carrying documents, can effectively identify the unidentified objects, and can re-position the bleaching identity personnel by breaking through the limitations of the traditional pursuit means, and can fully depict the working objects. The technology also can provide real-time monitoring and control for important personnel, can help the lost elderly to find family members, can provide verification services for the Internet + police, and can facilitate the masses to ask for help online [4].

At present, Yitu Technology has reached cooperation with the Ministry of Public Security, China Border Inspection, China Marine Police and dozens of public security agencies, and has successfully completed security at major events such as the 2016 Hangzhou G20 Summit and the 2017 BRICS Leaders Summit. It's work has already unanimously recognized by all parties.

\subsection{Kuangshi technology Co., Ltd. and its intelligent security solutions}

Kuangshi technology corporation's intelligent security solutions based on face recognition technology, can be widely used in video surveillance, intrusion alarm, access control, access control, electronic patrol, network alarm and other major security areas, which can effectively help government security related agencies, including public security, customs, airports, railways identify suspicious person, certificate real name, set down face electronic fence and accuse key personnel.

Its products ,including portrait recognition series (sky system, massive portrait search system), video structured series (video structured engine, structured research and judgment system), pro-detection series (portrait temporary bayonet system, structured service box), vehicle identification system ( vehicle bayonet system and vehicle model evaluation platform) are widely used in three-dimensional security control, intelligent border inspection, intelligent prevention and control, public transportation, temporary deployment control, prison monitoring, military traffic management and vehicle management [5].

In the application scenarios of key places management and control, comprehensive treatment and group stability incidents, portrait bayonet retrofits, camp and perimeter security, three-dimensional prevention and control, etc., Kuangshi Technology ,relying on its high-speed modeling and storage, high-precision identification and second-level response, gains superior performance .

\subsection{Shangtang Technology Co., Ltd. and SenseFace face combat platform}

Shangtang Technology independently develops face static comparison server, face dynamic comparison server, face control actual combat platform, view intelligence research and judgment system, video structured analysis server, identity verification service, face recognition machine (gate machine version), etc. Services and products are gradually establishing close cooperative relations with local public security organs. One of successful case of cooperation is a public security bureau in Guangzhou City use the Shangtang view intelligence research and judgment system. The public security bureau in Guangzhou City use the search function of the map search to compare the face of the crime scene with the standard library photos, conduct the identity check of the personnel, and crack down on the crimes 
that have occurred. Since 2017, the video investigation department has successfully compare more than 2,000 people in the investigation, arrested more than 800 people, and successfully solved nearly 100 cases [6]. The Yunnan Provincial Public Security Department used the SenseFace face-to-face combat platform of Shangtang Technology to effectively assist the police to investigate illegal criminal activities and combat terrorist activities. At present, Shangtang Technology has built a test platform for the construction of face recognition platforms in various provinces of Yunnan Province. In the future, it will build a comprehensive application platform for provincial and departmental portrait recognition, which will help public security officers effectively stop the behaviors that endanger public order [7].

\subsection{Guangzhou pixel Co., Ltd. and its face recognition large-scale search comparison system}

Guangzhou Pixel Co., Ltd is dedicated to criminal detection face recognition, its face recognition verification products, such as smart face recognition gates, face/fingerprint identification and verification machine, ID face recognition verification system, visitor self-registration machine (vertical verification machine), face/fingerprint collection and verification mobile terminal and social security live detection face recognition verification system, etc., have been widely used in public security work and in many industries. Guangzhou Pixel Company's face recognition large-scale search comparison system is a real-time and efficient and comprehensive information intelligent interaction system. Over the years, it has assisted the Guangzhou Public Security Bureau in playing an important role in pursuit, case-solving, tracing, and control personnel.

In the field of dynamic monitoring face recognition, Guangzhou Pixel's products, such as, video face recognition analysis system, grid dynamic face recognition monitoring and analysis system, dynamic face recognition identity verification system, massive video face analysis system and face recognition video attendance. All of these products contain the function of high-definition video capture, compression, distribution and storage; face detection, tracking and posture analysis; face comparison, filtering and early warning mechanisms .By fast and accurate recognition ,these products assist Foshan Public Security Bureau and Guangzhou Public Security to crack many cases happened in crowded places such as subway buses [8].

\subsection{Jinshi Technology Co., Ltd and its face recognition service}

Compared with other face recognition companies, Jinshi Technology Co., Ltd (LinkFace) has three core advantages: ultra-large-scale databases, advanced algorithms for self-developed research, and super computing platforms. These three core strengths make it one to two orders of magnitude better than other companies that do face recognition in real-world scenarios. LinkFace provides the world's leading face detection and recognition technology services. It has been the world's number one in the FDDB face detection public test and the world's first in the 300-W Benchmark accuracy rate. The LFW face recognition accuracy rate has reached $99.5 \%$. above [9].

Although LinkFace mainly provides solutions for the financial industry, its leading original algorithms and excellent deep learning capabilities still make it a frequent visitor in the field of public security criminal investigation and related research. In addition, LinkFace's database often assists the research team of the Ministry of Public Security in the face recognition algorithm debugging, and is often used in the case of major cases.

\section{Prospect of Face Recognition Technology in Public Security}

At present, in terms of algorithm accuracy, face recognition technology mostly adds new rules on open source libraries such as open source OPENCV (deep learning for cascading operations), and the difference in recognition accuracy between companies is only at the decimal point. The products of each 
company are advanced to the application stage, mainly for the actual work, which needs to be optimized. In terms of algorithms, Yitu Technology Co., Ltd, Kuangshi Technology Co., Ltd and Shangtang Technology Co., Ltd are in a leading position, which mainly relying on a large database to provide accurate face recognition services, and these three companies repeatedly make meritorious deeds in major events [10]; in terms of terminals, Guangzhou pixels Co., Ltd and Haixin Kejin Co., Ltd has developed a series of practical end products by building the long-term cooperation with the public security system, which has promoted the wide application of face recognition products and played an important role in grassroots work. As a rising star, Jinshi Technology Co., Ltd has not yet translated the algorithm into a practical product, but its future can still be expected.

This paper focuses on face recognition technology and the main related products for China's public security criminal investigation work. It mainly introduces the principle and structure of the commonly used face recognition software, and conclude several face recognition technology in cooperation with public security criminal investigation on the market. The technology company's face recognition products and related products applicable to public security criminal investigation work were summarized and pointed out, and the characteristics of each product system and the popularity of the application were pointed out [11]. It should be pointed out that face recognition technology is only a part of artificial intelligence technology. There are also many identification technologies such as fingerprint recognition, DNA identification and speech recognition in the public security field. Therefore, it is necessary to combine various techniques and methods to form a combination punch, which means combine face recognition with fingerprint recognition and speech recognition .In this way, application efficiency and accuracy can be improved, compared with only rely on the technology of face recognition. In addition, it can also be combined with human dynamics, such as motion recognition based on the geometric angle of Kinect bone space, human behavior recognition based on three-dimensional spatiotemporal features ,and so on[12].

\section{References}

[1] Mao Liang, Li Lichen. On the face recognition technology [J]. China Public Safety, 2014(10).

[2] Ma Yaping. Deeply explore the value of video, and deeply understand the needs of users. Open the security vision with AI Interview with Wan Dingrui, General Manager of Shenzhen Shenwang Vision Technology Co., Ltd.[J]. China Security, 2017(9).

[3] Tian Shuang, Wei Wenyuan. Constructing Public Security Intelligent Monitoring, Early Warning and Control System with Artificial Intelligence [J]. China Security, 2017(8).

[4] Jing Chenkai, Songtao, Zhuang Lei. A Survey of Face Recognition Technology Based on Deep Convolutional Neural Networks [J]. Computer applications and software, 2018(1).

[5] Zou Zhihuang, Sun Xin, Cheng Wushan. Overview of the development of face recognition technology products [J].Television technology, 2008, 32(1).

[6] Zhang Guangcheng, Zhang Guofei. Application of face recognition technology in the field of public safety [J]. China Public Safety, 2017(7).

[7] Wan Genxun, Li Jianyong, Qiang Tian, et al. Test system and test method of millions of one-to-many face recognition products:, CN $101615196 \mathrm{~A}[\mathrm{P}] .2009$.

[8] Hwang W, Kee S, Park G, et al. Face recognition method, and system using gender information: US, US 20070104362 A1[P]. 2007.

[9] Phillips P J, Flynn P J, Scruggs T, et al. Overview of the Face Recognition Grand Challenge[C]// IEEE Computer Society Conference on Computer Vision and Pattern Recognition. IEEE Computer Society, 2005:947-954.

[10] Keers R N, Williams S D, Cooke J, et al. An Overview of Holistic Face Recognition [J]. Ijrcct, 2013, 2(9):738-741.

[11] Căleanu C D. Face expression recognition: A brief overview of the last decade[C]// IEEE, International Symposium on Applied Computational Intelligence and Informatics. IEEE, 2013:157-161.

[12] Meethongjan K, Mohamad D. A Summary of literature review: Face Recognition [J]. Transactions of the Japan Society of Mechanical Engineers A, 2007, 2007:3-4. 